\title{
Erratum to: Open questions and novel concepts in oral cancer surgery
}

\author{
Giancarlo Tirelli $^{1} \cdot$ Serena Zacchigna $^{2} \cdot$ Matteo Biasotto $^{3} \cdot$ Marco Piovesana $^{1}$
}

Published online: 2 July 2015

(c) Springer-Verlag Berlin Heidelberg 2015

\section{Erratum to: Eur Arch Otorhinolaryngol \\ DOI 10.1007/s00405-015-3655-0}

The original version of this article unfortunately contained a mistake. Figures 1 and 2 were interchanged. The correct version of the figures is given below. The original article has been amended accordingly.

The online version of the original article can be found under doi:10.1007/s00405-015-3655-0.

\section{Marco Piovesana}

marcopiovesana81@gmail.com

1 Division of Otolaryngology and Head and Neck Surgery, Cattinara Hospital, University of Trieste, Trieste, Italy

2 Molecular Medicine, International Centre for Genetic Engineering and Biotechnology (ICGEB), Trieste, Italy

3 School of Dental Sciences, University of Trieste, Trieste, Italy 


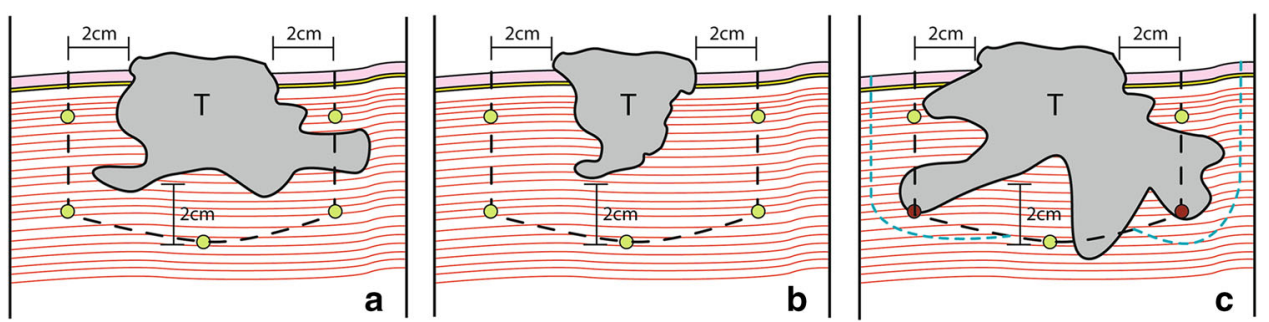

- Resection Line

- - - Intraoperative

Resection Extension

- Positive Frozen Section

- Negative Frozen Section

Fig. 1 In an attempt to achieve clear margins, while resecting OSCCs with a similar superficial extension, the surgeon might face the following paradigmatic situations: a incomplete resection despite all intraoperative frozen sections being free from disease; $\mathbf{b}$ complete

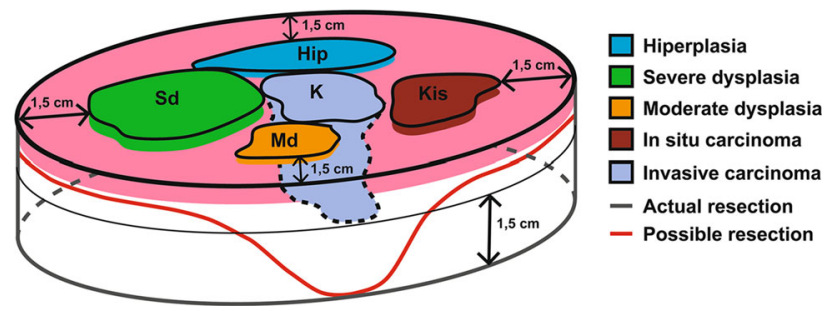

Fig. 2 Potential surgical approach using in vivo monitoring of the tumor invasion pattern. We can observe a remarkable sparing of uninvolved tissue resection with all intraoperative frozen sections free from disease; c incomplete resection despite lateral extension of the resection line due to positive intraoperative frozen sections 